\title{
Evaluando el \\ rendimiento en la tabla de golpeo del makiwara
}

Paul K. Smith ${ }^{1}$, Timothy Niller 2 , \& Peter W. McCullough ${ }^{3}$

${ }^{1}$ W est Chester University of Pennsylvania (EE.UU.), ${ }^{2}$ Penn State Brandywine (EE. UU.), 3Upper Darby High School (EE.UU).

Es traducción del artículo Evaluating Makiwara Punching Board Performance, publicado en el Journal of A sian Martial Arts, volumen 19, número 2 (34-45), 2010

\section{Resumen}

No hay en la literatura estudios que cuantifiquen las características del impacto en las tablas del makiwara. Lógicamente, la evaluación de las características mecánicas de varios diseños de tablas de makiwara debería ser un paso inicial en la determinación de la validez del makiwara como medio de desarrollo de la técnica del kárate. Consecuentemente, el propósito de este estudio fue determinar la dureza de los diseños del makiwara que se estrecha y en tabla apilada, utilizando técnicas estáticas de carga para evaluar su idoneidad para practicantes de diferentes niveles. Cuando nuestros resultados se observan en términos de progresión del entrenamiento, parece que el diseño apilado de fresno, más flexible, se ajusta mejor al principiante, mientras que el modelo que se estrecha de encina, más rígido, se ajusta mejor al experto.

Palabras clave: Kárate, entrenamiento, técnica, fuerza, materiales, diseños.

\section{Evaluating Makiw ara Punching Board Performance}

Abstract: No studies quantifying the impact characteristics of makiwara boards are available in the published literature. Logically, an initial step in determining the validity of the makiwara to develop karate technique would be to assess the mechanical characteristics of various types of makiwara board designs. It was, therefore, the purpose of this study to determine the stiffness of selected tapered board and stacked board makiwara training devices using static loading techniques to assess their suitability for practitioners of different levels. When our results are viewed in terms of progression of training, it would appear that a novice would be more suited to the more compliant ash stacked design and the expert more suited to the stiffer oak tapered design.

Key words: Karate, training, technique, strength, materials, designs.

Avaliando o rendimento do golpe na tábua de makiwara

Resumo: Não existem estudos que quantifiquem as características do impacto nas tábuas de makiwara. Logicamente, a avaliação das características mecânicas de vários desenhos de tábuas de makiwara deveriam ser um passo inicial na determinação da validade do makiwara como meio de desenvolvimento da técnica de karaté. Consequentemente, 0 propósito deste estudo foi determinar a dureza dos desenhos de makiwara, utilizando técnicas estáticas de carga para avaliar a sua idoneidade para praticantes de diferentes 
níveis. Quando os nossos resultados se observam em termos de progressão de treino, parece que o desenho empilhado de fresno, mais flexível, se ajusta melhor ao principiante, enquanto o modelo simples de azinheira, mais rígido, se ajusta melhor ao praticante avançado.

Palavras-chave: Karaté, treino, técnica, força, materiais, desenhos.

El Dr. Paul Smith GOLPEANDO LA TABLA DEL MAKIW ARA

Ilustraciones cortesía de

P. Smith excepto si se indica lo contrario.

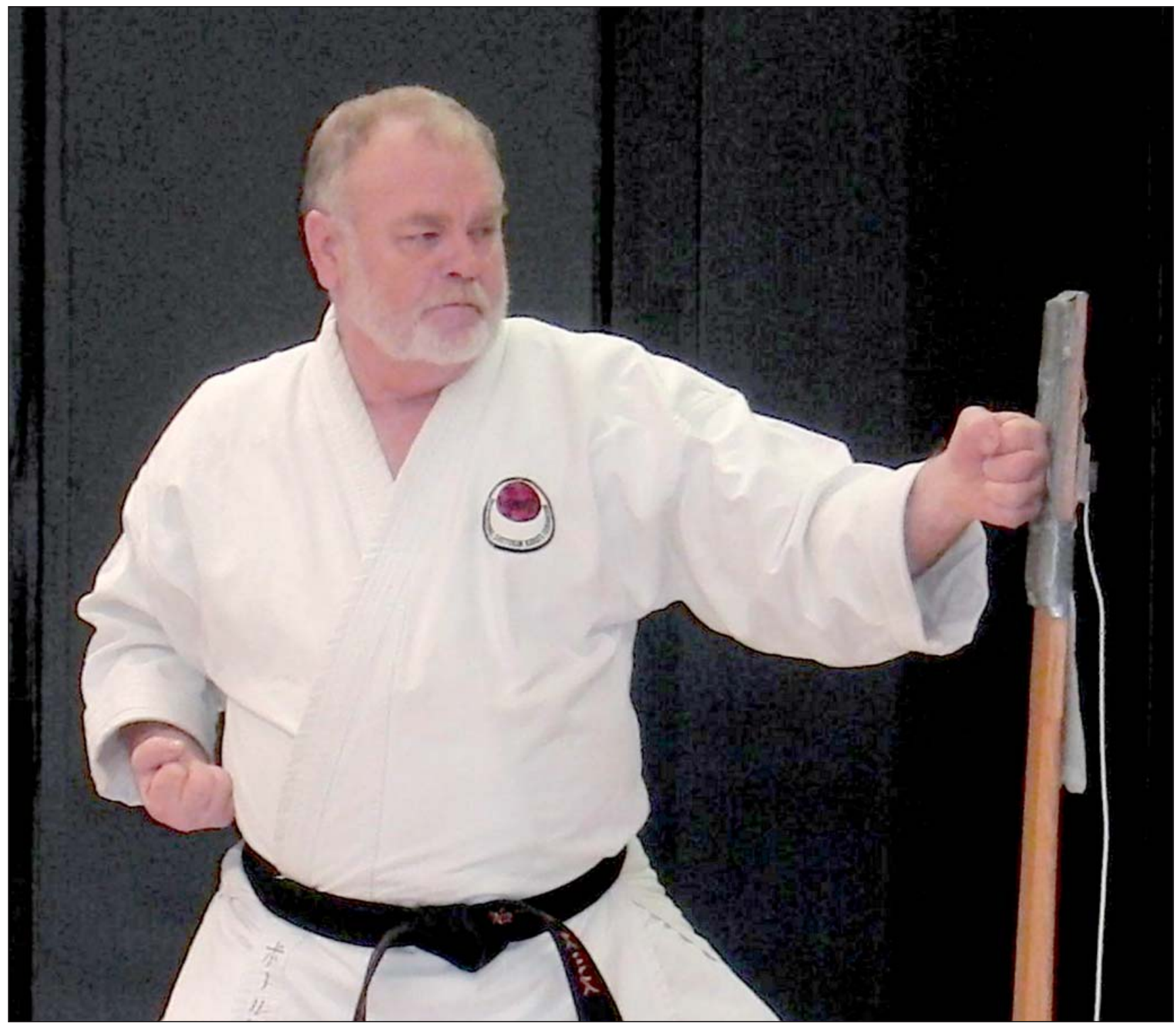




\section{Evaluando el \\ rendimiento en la tabla \\ de golpeo del makiwara}

Paul K. Smithin ${ }^{1}$, Timothy Niller² 2 \& Peter W. McCullough ${ }^{3}$

${ }^{1}$ W est Chester University of Pennsylvania (EE.UU.), ${ }^{2}$ Penn State Brandywine (EE. UU.), 3Upper Darby High School (EE.UU).

\section{Introducción}

El kárate es un arte marcial japonés que brinda al practicante los beneficios del entrenamiento en autodefensa, además del desarrollo de la salud, psicomotricidad, cognición y afectividad. Como tal, es un excelente medio para la educación física, además de su valor añadido para el aprendizaje de habilidades prácticas. En el entrenamiento del kárate, los puñetazos y las patadas son los dos tipos de técnicas más habituales para vencer al oponente. El principio de "un golpe, una muerte" (ikken hitsatsu) es característico de ambos, un concepto japonés que significa que el practicante debería ser capaz de inhabilitar a un atacante con un golpe (Okazaki \& Stricevic, 1984) -no significa que literalmente tenga que "matar" al oponente-. De los estimados 50 millones de personas que entrenan kárate (Smith, 1984), es probable que cualquiera de ellos esté, en algún momento, expuesto al fenómeno de golpear algún tipo de aparato para mejorar su habilidad y tener sensaciones" de lo que es impactar sobre un objeto. El entrenamiento del makiwara ("paja enrollada", tabla de golpeo) es considerado por muchos defensores de este arte marcial tradicional como un elemento integral para el desarrollo de una correcta técnica de golpeo, hasta alcanzar los correctos estándares de calidad (O kazaki \& Stricevic, 1984).

La tabla del makiwara es un aparato montado verticalmente, fijado o enterrado en un extremo (Figura 1), que se utiliza para desarrollar la efectividad de los puñetazos por medio de golpeos repetidos a una almohadilla sujeta al extremo libre de la tabla. La almohadilla es normalmente de alguna variedad de espuma, cuerda, paja 0 material textil que sirve para suavizar el impacto en los nudillos. La forma de la tabla puede ser estrechada ${ }^{1} 0$ apilada ${ }^{2}$, y sus dimensiones son generalmente de $8,89 \mathrm{~cm}$ (3,5 pulgadas) de anchura por $127 \mathrm{~cm}$ (50 pulgadas) de

\footnotetext{
1 Las tablas de makiwawa que se estrechan son fabricadas en una única pieza de madera más gruesa en su base y que se estrecha hacia la parte superior, donde se sujeta la almohadilla de golpeo. Son más difíciles de construir ya que para su fabricación se necesitan tornos y otros instrumentos específicos de carpintería.

2 Las tablas de makiwara apiladas constan de varias tablas individuales cortadas con la misma anchura y grosor pero distintas longitudes. En la base se sujetan juntas para dar firmeza, con la tabla más corta al final y la tabla más larga delante, frente al practicante.
} 


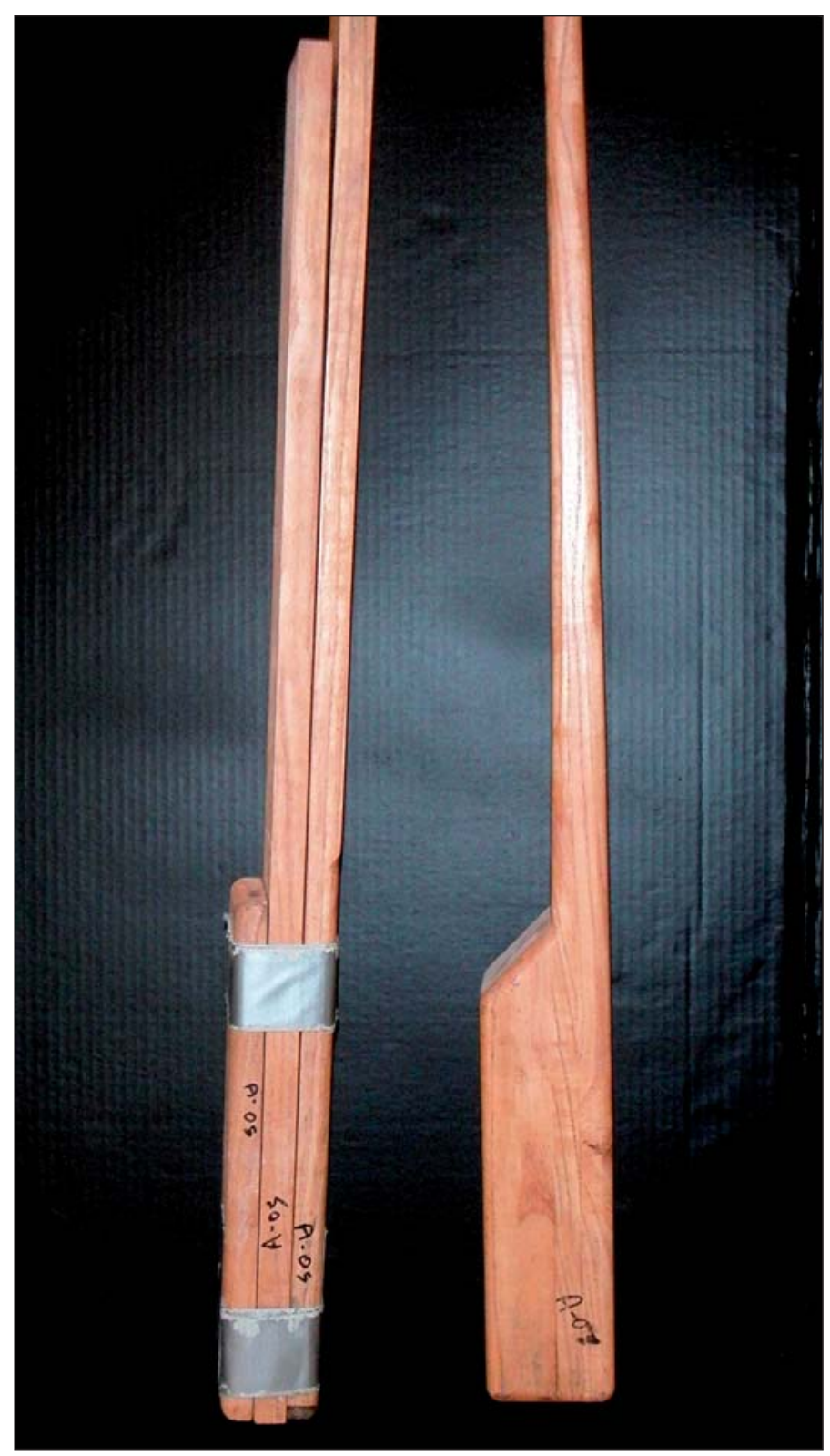

altura sobre el suelo. Los principios físicos generales implican que el tipo de madera utilizada para la tabla, su longitud y grado de estrechamiento (del suelo al extremo superior) deberían determinar la fuerza del "muelle" del aparato. Otro modelo habitual consiste en varias tablas de diferente longitud apiladas en orden descendiente para crear una configuración de lámina-muelle. También se han utilizado diversos tipos de maderas, duras y blandas. Normalmente, el estudiante escoge las tablas con las que entrenar en función de sus preferencias personales 0 de las tablas que estén disponibles en la sala de entrenamiento (O kazaki, 1998).

El mecanismo por el cual la tabla ayuda a desarrollar la eficacia de golpeo implica la coordinación de los movimientos corporales, de tal modo que la "fuerza" o "energía" se concentre o dirija hacia un punto concreto del objetivo. Este objetivo se encuentra normalmente a 5,08-15,24 cm ( 2 a 6 pulgadas) detrás de la superficie de golpeo, y requiere coordinar los impulsos parciales de los segmentos de la persona que golpea para impactar con su "arma" (puño o pie), que recibe la inercia del resto del cuerpo, moviéndose a una velocidad óptima en el momento del impacto. Para detener a un asaltante se necesitan impactos de distintas características, dependiendo de qué parte del cuerpo esté implicada. Los impactos pueden clasificarse en función de la relación masa-velocidad, como "alta velocidad/masa pequeña" o "baja velocidad/ masa grande", y en función de la naturaleza del movimiento del "arma" que golpea, e.g., aplicación lineal 0 angular de la fuerza. El puñetazo se

FIGURA 1: FOTOS DE LAS TABLAS DE MAKIW ARA APILADAS Y ESTRECHADAS.

EL DISEÑO DE MAKIWARA APILADO CONSTA DE VARIAS MADERAS QUE ESTAN SUJETAS

JUNTAS, MIENTRAS QUE

EL DISEÑO DEL MAKIWARA

ESTRECHADO CONSTA DE UNA SOLA PIEZA DE MADERA MOLDEADA. considera como una técnica de relativamente baja velocidad/masa grande, en la que la fuerza se aplica de un modo lineal (Smith et. al., 1993). Brazo y antebrazo son lanzados a modo de "empujón" hacia el objetivo, en vez de como un látigo 0 a modo de snap, en una acción como de "lanzamiento" (Kreighbaum \& Barthels, 1996). Este tipo de técnica de "empujón" se utiliza preferiblemente para golpear el tronco del oponente, donde se necesita penetrar más profundamente en un tejido relativamente blando, o sobre huesos vulnerables, para romper, acelerar o lacerar los huesos, nervios y tejidos del cuerpo. Por su parte, la acción de "lanzamiento", conocida comúnmente como técnica de percusión o snap, se utiliza para atacar zonas más duras o débiles del cuerpo, tales como la nariz o las articulaciones, y "causar shock" en los tejidos impactados bajo la superficie o romper los relativamente frágiles tejidos duros de huesos 0 articulaciones. 
De acuerdo con Smith (1999), la técnica apropiada para golpear el makiwara sería estar de pie justo en frente de la tabla en la postura elegida y aplicar la técnica "golpeando a través" de la almohadilla de la tabla, manteniendo momentáneamente toda la penetración para permitir que el cuerpo entrene los músculos y "enfoque", o concentre la energía, al final del movimiento. La fuerza de golpeo debería aplicarse suavemente al principio y progresar hacia golpes más fuertes a medida que se gana experiencia. El cuerpo se adaptará a las fuerzas de reacción o carga de la tabla. Se debería poner atención en penetrar en la superficie del objetivo hasta la máxima extensión de la técnica, y no "abofetear" la tabla. Esto permite al cuerpo coordinar y desarrollar el sistema neuromuscular para lograr una ejecución de las técnicas más efectiva.

No se ha publicado ninguna secuencia específica de entrenamiento, o periodización (Smith, 1999), que indique el número de series y de repeticiones por serie para el entrenamiento con makiwara. Sobre la base de los principios de progresión del entrenamiento (McArdle, Katch \& Katch, 2001) y la experiencia práctica de los autores, parece razonable comenzar con un número bajo de series y repeticiones y progresar hacia un número superior tanto de repeticiones como de cargas. Un entrenamiento similar, progresivo de fuerza, ha mostrado ser eficaz en la mejora del rendimiento de las patadas en practicantes de taekwondo (Jakubiak $\&$ Saunders, 2008).

\section{Método}

Un primer paso para determinar la eficacia de las tablas de makiwara para el entrenamiento de fuerza es evaluar las propias características de dureza de la tabla, sin intervención humana. Esto puede realizarse a través de la técnica de carga-desviación, en la que la tabla del makiwara se carga con varios pesos conocidos mientras se mide la desviación resultante. La carga y la desviación pueden representarse en un gráfico, de modo que la dureza quedaría definida como la inclinación de la curva resultante. Así, los materiales con curvas de carga-desviación más pronunciadas son más rígidos y resisten la deformación con mayor facilidad. Entendiendo esto, es tentador proceder, observar la dureza de una determinada madera, y sacar conclusiones sobre qué madera sería más adecuada para el nivel de entrenamiento del practicante. Un usuario de makiwara experto escogería una madera más dura que un principiante. Sin embargo, este planteamiento pasa por alto algunas cuestiones clave. Primero, la dureza por sí misma no dice nada sobre la cantidad de fuerza que se necesita para doblar una tabla de una determinada forma 0 estructura. Un experto tendrá las mismas dificultades que un novato al golpear un travesaño de madera fijo de $10 \times 10 \mathrm{~cm}$, independientemente del tipo de madera de la que esté hecho este travesaño, ya que esta geometría tiene poca flexibilidad. Por tanto, también es necesario comprobar realmente las formas, con el objeto de establecer las cotas de fuerza tanto para la madera como para el diseño. Segundo, es necesario realizar muchas comprobaciones de cada tipo de madera y diseño para asegurar la fiabilidad de los resultados. La madera es bastante variable por naturaleza, y aspectos como la edad, fibra y otros factores pueden provocar diferencias en la dureza. 


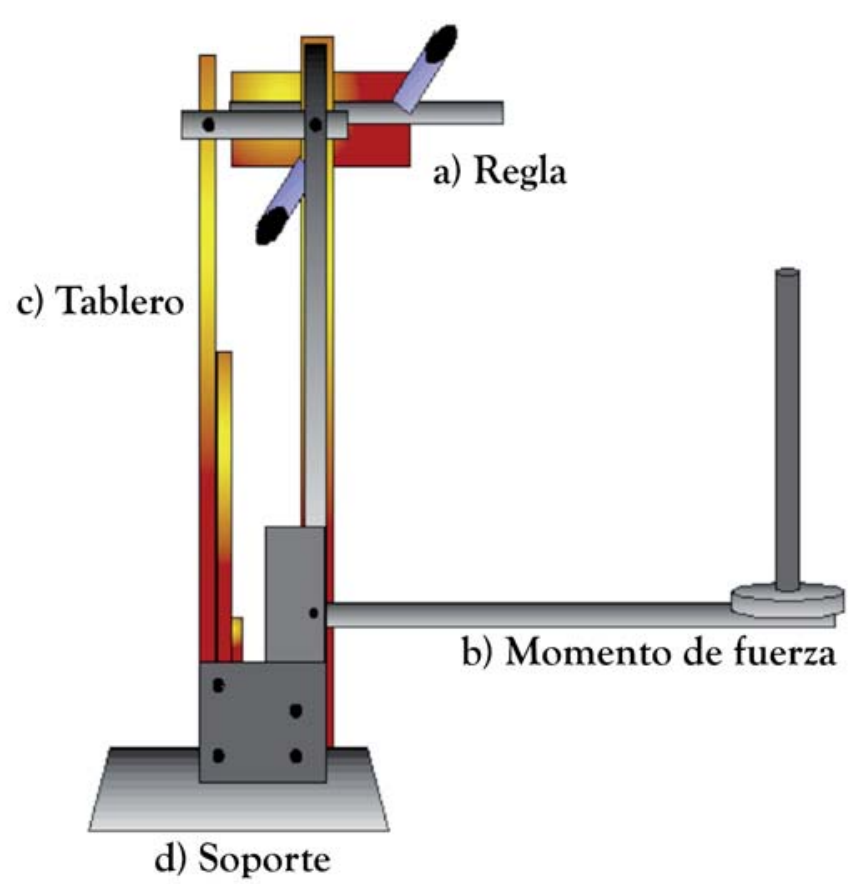

Figura 2: El DISPOSITIVO Del MAKIW ARA. Se SUJETÓ UNA REGLA (A) PERPENDICULAR A LA TABLA DEL MAKIWARA (c) PARA DETERMINAR LA DESVIACIÓN.

EL DISPOSITIVO DE CARGA

CONSTABA DE UN BRAZO

DE FUERZA (B) DONDE SE PODÍAN COLOCAR LOS PESOS PARA DESVIAR LA TABLA. EL

SOPORTE (D) SE INSTALÓ EN HORMIGÓN UTILIZANDO TIRAFONDOS PARA ASEGURAR LA INMOVILIDAD DE LA BASE.
Teniendo en cuenta estas consideraciones, se construyeron cinco reproducciones de tablas de makiwara de fresno blanco del norte, cerezo de Pensilvania, roble rojo y abeto Douglas, con dos diseños (estrechado y apilado), para comprobarlos en un mecanismo de carga creado específicamente para este propósito (Figura 2). Las tablas fueron analizadas cargando cada una de ellas con pesos y grabando los valores de desviación, en incrementos de $111.2 \mathrm{~N}$ (25 libras) de peso y con un rango desde $111.2 \mathrm{~N}$ hasta $1000.8 \mathrm{~N}$ (25-225 libras), en orden compensado para distribuir cualquier efecto de estrés acumulado durante el estudio. Los datos de carga-desviación fueron representados para cada especie y diseño de tabla. Además, se representaron las líneas optimizadas para mostrar las tendencias medias en la dureza para cada tipo de tabla, con el objeto de facilitar la comparación visual entre las maderas y diseños.

Este método de visualizar los resultados tiene una desventaja desde el punto de vista científico: a pesar de las tendencias visuales uno no puede decir si las diferencias en las pendientes de las curvas son significativas 0 no. Cuando decimos que existe una diferencia significativa entre dos grupos, quiere decir que cualquier diferencia observada entre los grupos no es el resultado de una sola probabilidad aleatoria. Sin significatividad estadística, solemos decir que el resultado es anecdótico y no tenemos confianza de que los resultados sean repetibles. La herramienta estadística utilizada en estos casos es el análisis de la varianza (AN 0 VA), que determinará si existe alguna diferencia significativa entre los diseños y/o especies de madera. Dado que tal diferencia existe, un test post-hoc identifica específicamente qué grupos son diferentes al resto.

\section{Resultados}

Los resultados generales se resumen en las Tablas 1 , 2 y 3. La Tabla 1 presenta los resultados en forma de las medias combinadas de desviación ( $\mathrm{mm}$ ) de la tabla de makiwara de las especies de fresno, cerezo, abeto y roble para los tipos de tablas apiladas (TA) y estrechadas (TE). Las medias de desviación $(\mathrm{mm})$ para las tablas de makiwara apiladas se muestran por especie en la Tabla 2 mientras que las medias de desviación para las tablas que se estrechan se muestran en la Tabla 3. Las especies de madera se han abreviado como fresno $(F)$, cerezo $(C)$, abeto $(A)$ y roble $(R)$. Las diferencias en el número de tablas $(\mathrm{N})$ de cada especie se deben a la rotura. Nuestra utilización de una ANOVA 
2x4 (dos tipos de tablas por cuatro tipos de madera) con unos tests post-hoc de Neuman-Keuls indicaron que existía, de hecho, una diferencia significativa entre las propiedades de fuerza-desviación en todos los grupos excepto para las tablas que se estrechan de fresno y cerezo.

\section{Discusión}

Una investigación más profunda de los resultados indicó varias tendencias dignas de atención. Las tablas estrechadas mostraron una flexión significativamente menor ante la carga que las tablas apiladas (Tabla 1). Esto no es sorprendente ya que entre las tablas apiladas no existe otra fuerza horizontal de sujeción más que la de fricción. Esto permite que, ante una carga, las tablas apiladas se deslicen entre sí, a diferencia de las tablas que se estrechan que no tienen este grado de libertad. Por tanto, existe una mayor movilidad de la superficie de golpeo en el diseño apilado. Una consecuencia de esta movilidad es que las tablas apiladas se aproximarán más rápidamente a sus límites elásticos, más allá de los cuales no regresarán a su forma original o se partirán. En realidad, la rotura ocurrirá con bastante frecuencia en las tablas apiladas más débiles. Específicamente, se rompieron cuatro tablas apiladas de abeto (80\%), cinco de fresno (100\%), y dos de cerezo (40\%). No se rompió ninguna de las tablas apiladas de roble, y tampoco se rompió ninguna tabla con la carga menor (111.2 N).

\begin{tabular}{cccc} 
TA BLA 2 & & & \\
\hline Madera & N & Media & Desv. Típ. \\
C & 41 & 118 & $74^{*}$ \\
F & 36 & 106 & $71^{*}$ \\
A & 34 & 94 & $64^{*}$ \\
R & 45 & 90 & 59 \\
\hline & & & $* p<0,05$
\end{tabular}

\begin{tabular}{cccc} 
TABLA 3 & & & \\
Madera & N & Media & Desv. Típ. \\
C & 45 & 78 & $54^{*}$ \\
F & 45 & 76 & $52^{*}$ \\
A & 45 & 55 & $36^{*}$ \\
R & 45 & 50 & 33 \\
\hline & & & $* p<0,05$
\end{tabular}

\section{TABLA 4}

Tipo de tabla

\begin{tabular}{ccc} 
Madera & Estrechada (N/m) & A pilada ( N / $\mathbf{m})$ \\
\hline C & $5389 \pm 10$ & $3907 \pm 19$ \\
F & $5253 \pm 9$ & $3245 \pm 6$ \\
A & $8096 \pm 4$ & $3985 \pm 11$ \\
R & $8653 \pm 7$ & $4882 \pm 13$ \\
\hline
\end{tabular}

\section{Tabla apilada}

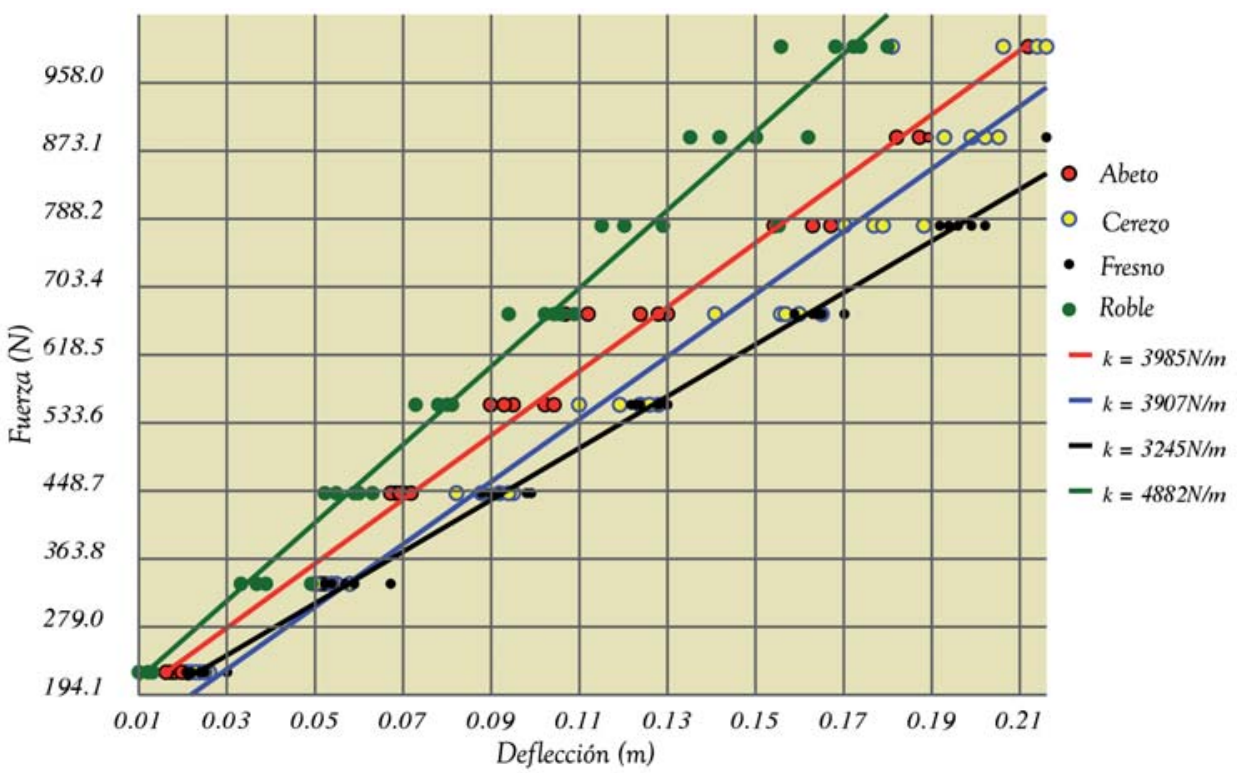

Figura 3: CuRvas de CARGA-DESVIACIÓN PARA LAS TABLAS APILADAS. LAS TABLAS QUE SE DESVÍAN MENOS CON UNA CARGA DADA SON CONSIDERADAS MÁS DURAS. LAS DIFERENCIAS SIGNIFICATIVAS EN LA FUERZA MEDIA Y DESVIACIÓN MEDIA FUERON IDENTIFICADAS ENTRE CADA TIPO DE TABLA APILADA. 
Figura 4: CurVa de CARGA-DESVIACIÓN PARA LAS TABLAS ESTRECHADAS. LAS DIFERENCIAS SIGNIFICATIVAS FUERON IDENTIFICADAS PARA CADA TIPO DE TABLA ESTRECHADA EXCEPTO ENTRE FRESNO Y CEREZO $(P<0.05)$.
Cuando se analizaron las tablas en función de la especie, tanto en el diseño estrechado como apilado, el roble tuvo la media de desviación más pequeña, seguido por el abeto, cerezo y fresno (Tablas 2-3). Esto es contradictorio con la media de los parámetros de flexión que ofrecen las tablas de materiales, en que uno esperaría que el fresno fuese el más duro y el abeto fuese el más flexible (Green et al., 1999). Sin embargo, ya que el parámetro de flexión del impacto puede variar hasta un $25 \%$ en una determinada tabla, los resultados son realistas. Además, la similitud de los resultados de una tabla a otra de la misma especie se debe a que estas tablas habían sido fabricadas a partir del mismo árbol. La dureza calculada a través de las líneas de carga/desviación optimizadas (Figuras 3-4) muestra las mismas tendencias que las desviaciones medias, siendo el roble la madera más dura y el fresno la más flexible (Tabla 4).

\section{Tabla estrechada}

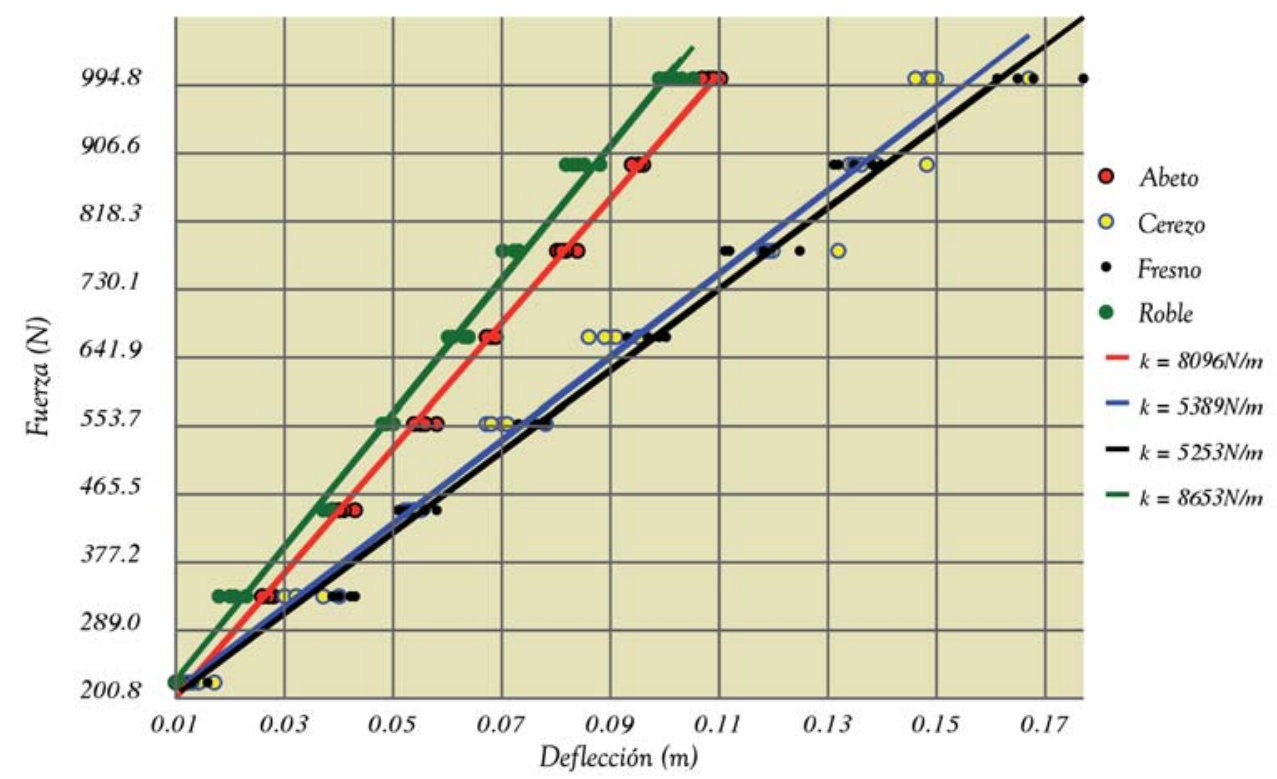

\section{Implicaciones para el entrenamiento}

Al observar este estudio desde la perspectiva de las famosas recomendaciones de incremento progresivo de la carga en el entrenamiento deportivo (McArdle, Katch $\&$ Katch, 2001), los resultados sugieren una facilitación para la progresión del entrenamiento y su correcto desarrollo. La menor dureza (la mitad) de las tablas apiladas implica su gran idoneidad para un practicante novato, ya que estas ofrecen menos resistencia y menores fuerzas de reacción al puñetazo. Para un entrenamiento realmente progresivo, uno podría empezar con el tipo de madera más débil (fresno) y avanzar hacia la más dura (roble). Sin embargo, respecto a la rotura de todos los tipos de madera de las tablas apiladas excepto el roble, quizás sólo el roble debería considerarse como material para este diseño. Aunque el roble tiene una dureza mayor, esta podría regularse cambiando el grosor de las tablas 0 añadiendo un almohadillado extra en la superficie de golpeo. Los practicantes 
más avanzados podrían luego avanzar al diseño estrechado para aumentar el entrenamiento de fuerza.

Este estudio también proporciona, por medio de la cuantificación de las curvas de fuerza-desviación de la tabla del makiwara, un procedimiento estándar para estimar la carga de trabajo asociada a un régimen determinado de práctica.
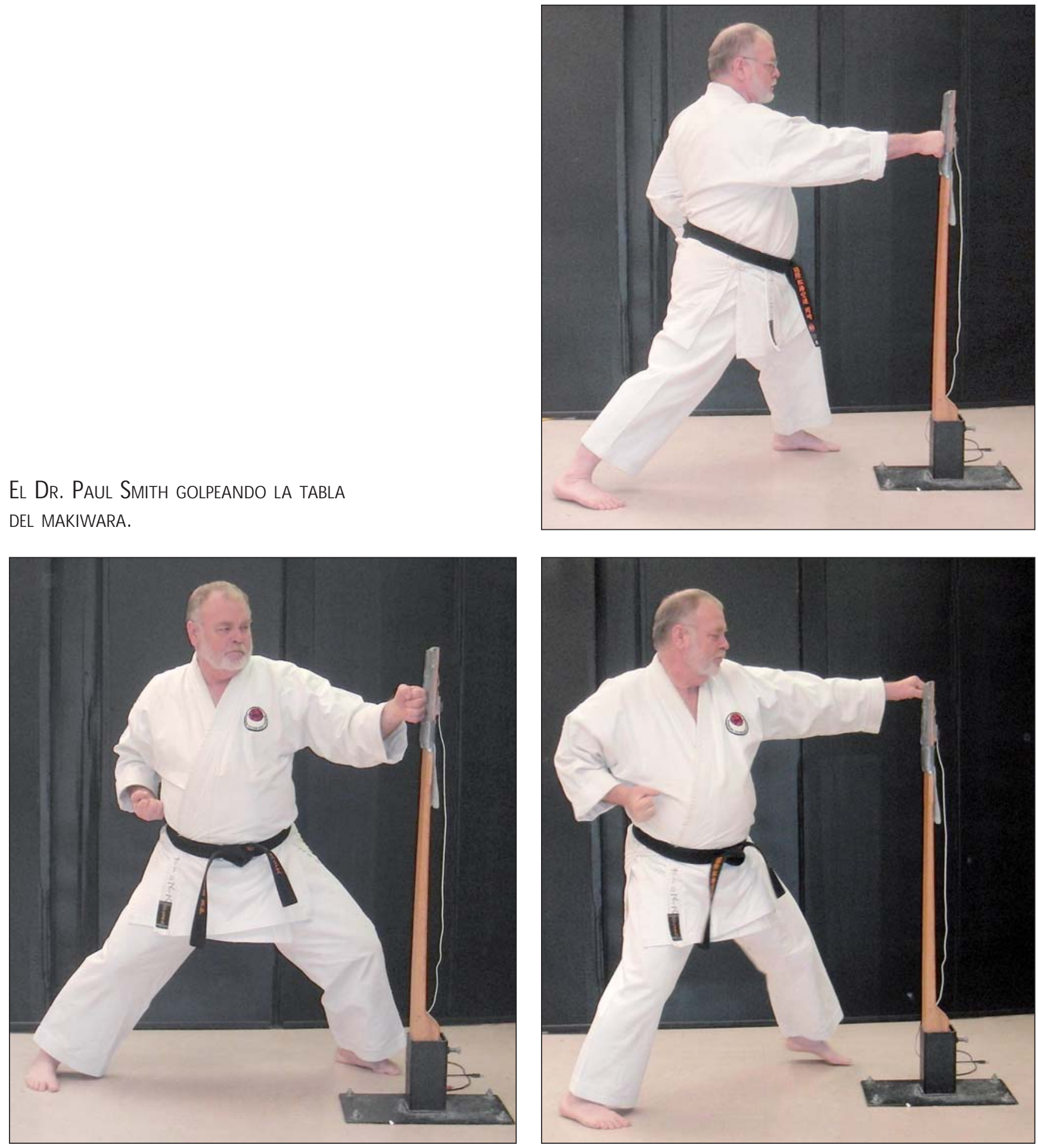

Smith, Niiler, \& McCullough $\diamond$ Evaluando el rendimiento en la tabla de golpeo del makiwara Revista de Artes Marciales Asiáticas $\diamond$ Volumen 5 Número 2 (111-122) $\diamond 2010$ 
llustraciones cortesía de

istockphoto.com
El American College of Sports Medicine (Colegio Americano de Medicina del Deporte) (ACSM) publica regularmente un estudio titulado "Modelos de Progresión en el Entrenamiento de la Fuerza", que es un resumen de la mejor práctica actual en el diseño de sesiones de entrenamiento de fuerza. De acuerdo con la actualización más reciente de este documento (ACSM, 2009), el entrenamiento de fuerza para principiantes debería ser del $60-70 \%$ de una repetición máxima (1RM) en un ejercicio dado. Un ejercicio normal que es parecido al golpe inverso es el press de banca. Si uno puede levantar 225 libras (102 kg) en 1RM (utilizando dos brazos), la mitad de esto sería 112.5 libras $(51 \mathrm{~kg})$. El $70 \%$ de la carga máxima para un brazo es 78.75 libras $(35,7 \mathrm{~kg})$ o 350N. De esta manera, si uno estuviera utilizando el makiwara apilado de cerezo, más flexible, la desviación correspondiente a esta carga sería de $6 \mathrm{~cm} 0$ aproximadamente 2.3 pulgadas. Considerando que el puñetazo es en realidad dinámico, sufriría una desviación mayor, pero esta estimación fija el límite inferior de lo que se podría esperar de tal persona. Alternativamente,

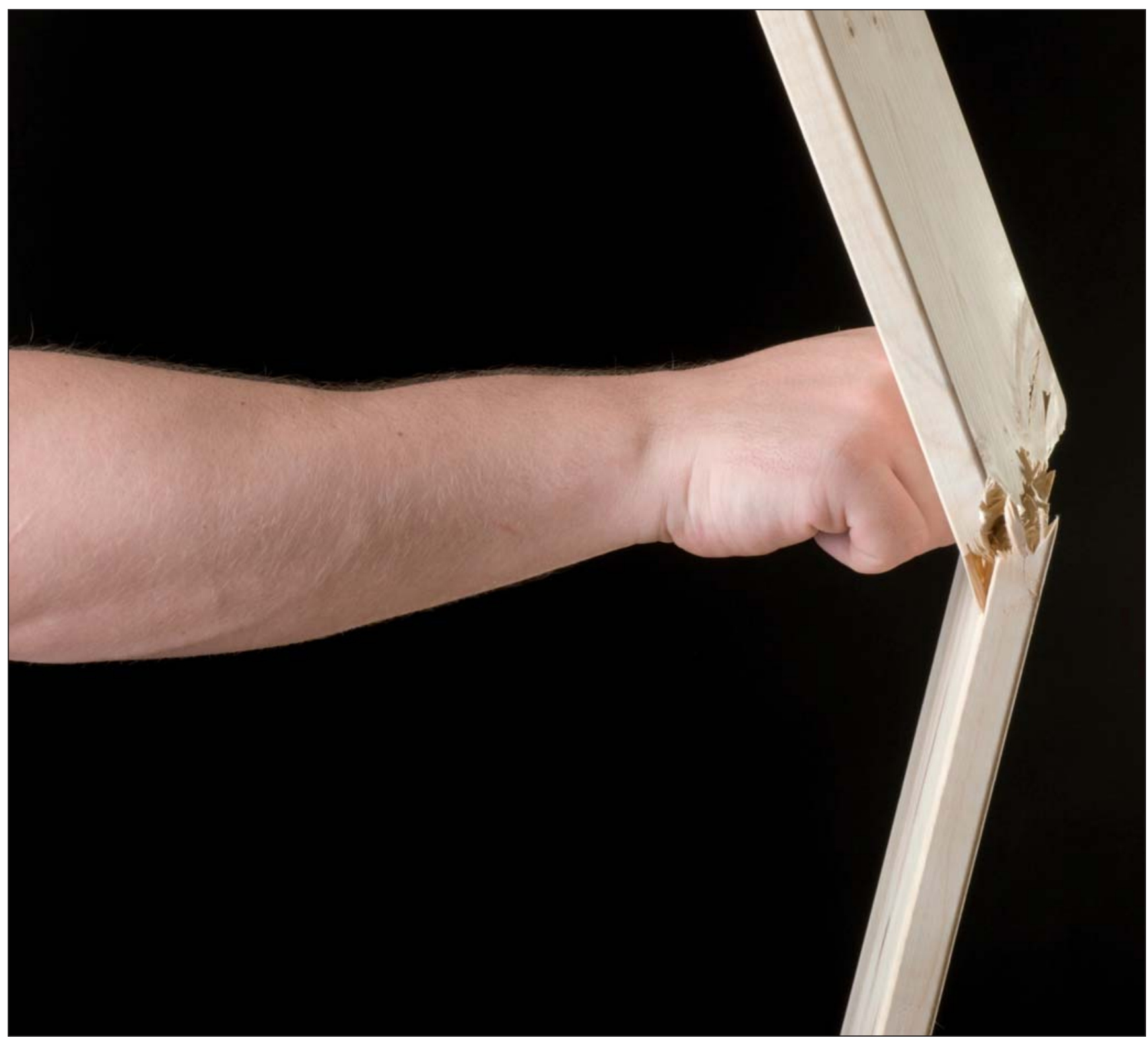


este cálculo podría invertirse para determinar la tabla de makiwara más apropiada para un individuo sobre la base de 1RM de su press de banca.

Habiendo establecido una forma para estimar la carga, entonces es posible aplicar las directrices del ACSM de acuerdo a las repeticiones para un entrenamiento progresivo. Aunque existen muchos protocolos diferentes, el generalmente citado de 8-12 repeticiones con múltiples series parece ser todavía el estándar para principiantes (ACSM, 2009). El reciente meta-análisis de 37 estudios sobre entrenamiento de fuerza indica que las ganancias en fuerza se optimizan con 8 series por grupo muscular (Peterson et al., 2005). Sin embargo, debido a que no se han realizado estudios sobre la eficacia de ese protocolo de ejercicio respecto a la tabla de makiwara, puede ser que un incremento de las repeticiones por serie y menos series pueda ser igualmente productivo.

\section{Conclusiones}

Puede concluirse que nuestros resultados mostraron diferencias entre los diseños de makiwara y entre los tipos de madera analizados. El diseño de tabla estrechado fue más duro que el apilado; sin embargo no existía diferencia entre el makiwara de cerezo y fresno en el diseño estrechado. La dureza del makiwara era progresivamente menor desde las tablas de madera de roble hasta las de abeto, fresno y cerezo. Una progresión lógica para el entrenamiento sería comenzar con el diseño apilado de una de las maderas de menor dureza a un makiwara hecho con el diseño estrechado de una de las maderas más duras. Sobre la base de las curvas de fuerza-desviación también es posible estimar aproximadamente la carga, y de este modo diseñar un programa efectivo para el entrenamiento de la fuerza.

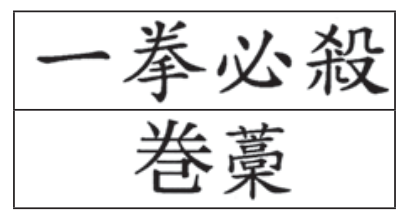

\begin{tabular}{|c|c|}
\hline ikken hitsatsu & "un golpe, una muerte" \\
\hline makiwara & "paja enrollada" \\
\hline
\end{tabular}

\section{REFEREN CIAS}

American College of Sports Medicine (Marzo de 2009). American College of Sports Medicine position stand. Progression models in resistance training for healthy adults. Medicine and Science in Sports and Exercise, 41(3):687-708.

Green, D., W inandy, J., \& Kretschmann, D. (1999). Wood handbook: Wood as an engineering material. Madison, W I: USDA Forest Service, Forest Products Laboratory. General technical report FPL; GTR-113: pp. 4.1-4.45.

Kreighbaum, E., \& Barthels, K. (1996). Biomechanics: A qualitative approach for studying human movement (4a $\mathrm{Ed}$.). N eedham Heights, MA: Allyn and Bacon. 
McArdle, W., Katch F., \& Katch V. (2001). Exercise physiology: Energy, nutrition and human performance. Baltimore, MA: Lippincott, W illiams \& W ilkins.

O kazaki, T. (1998). Comunicación personal. ISKF Headquarters Dojo, 222 S. 45th St., Philadelphia, PA, 19104.

O kazaki, T. \& Stricevic, M. (1984). Textbook of modern karate. Tokyo: Kodansha International.

Peterson, M., Rhea, M., \& Alvar, B. (2005). Applications of the dose response for muscular strength development: A review of metaanalytic efficacy and reliability for designing training prescription. Journal of Strength and Conditioning Research, 19: 950-8.

Stricevic, M., Dacic, D., Miyazaki, T., \& Anderson, G. (1989). Modern karate: A scientific approach to conditioning and training. Rockville Centre, NY: Miroto Karate Publishing Co.

Smith, P., Viano, D., Faust, D., \& Faust, L. (1993). Thoracic injury effects of linear and angular karate impact. En Biomechanics in Sports XI., Hamill, J., Derrick, T., \& Elliott, E. (Eds.), Amherst, MA: International Society of Biomechanics in Sports.

Smith, P. (1984). Selected impact characteristics of karate and boxing gloves. Unpublished doctoral dissertation. Southern Illinois University at Carbondale, Carbondale, IL. 